\title{
Cambio y desarrollo económico y político como resultado del capital democratico
}

José Gpe. Vargas Hernández - Ph.D. Instituto Tecnológico de Cd. Guzmán México.

\section{Resumo}

Este trabajo tiene por objetivo revisar los planteamientos teórico-metodológicos de la interacción dinámica entre las variables cambio económico y cambio político para la creación de capital democrático.

\section{Abstract}

The objective of this paper deals with the revisions over the questions on the theoretical dynamic interaction concerning on both several economic changes for creating the democratic capital.

\section{Palavras-chave}

Desarrollo económico, desarrollo político, capital democrático.

\section{Keywords}

Deconomic development, political development and democratic capital. 
José Gpe. Vargas Hernández

\section{INTRODUCCIÓN}

La construcción de una teoría del sistema del capitalismo democrático comprende una economía de mercado, una política democrática y un sistema cultural - moral que nutre los valores que son el sustento de las sociedades libres (NOVAK, 1993: 13-48). El sistema de libre mercado alienta el crecimiento económico la movilidad social. La libertad política introduce el pluralismo, la democracia, y la idea de un gobierno constitucional. El sistema moral-cultural se integra en las estructuras mediadoras de la familia, la iglesia y otras asociaciones voluntarias.

Los defensores argumentan que los principios del capitalismo democrático son teórica y prácticamente superiores a la visión socialista. El capitalismo democrático promueve la creatividad e iniciativa individual y se basa en el interés propio, no en el sentido del aprovechamiento egoísta individual sino en el beneficio a otros, siendo la familia el principal (YOUNKINS, 1999).

El estudio de la relación entre democracia y desarrollo económico ha sido uno de los aspectos más debatidos en la segunda mitad del siglo pasado. Para muchos analistas la democracia es la verdadera condición del desarrollo.Se presupone que la democracia estable promueve el desarrollo económico, el cual a su vez, promueve la consolidación del sistema democrático. Por lo tanto, la dinámica del cambio económico y el cambio político están conectados. El nivel de desarrollo económico tiene efectos positivos sobre el mantenimiento y consolidación de los sistemas democráticos (LIPSET, 1979).

No obstante, según la teoría de Rustow (1970), ningún nivel de desarrollo económico es prerrequisito para el establecimiento de instituciones democráticas sino más bien el sentimiento y compromiso de unidad nacional entre los diferentes estamentos sociales pero más significativamente, la disposición entre las elites para pactar acuerdos de transición.

El "movimiento de derecho y desarrollo" que se desarrolló en los setenta, analizó desde un etnocentrismo, la vinculación de los sistemas de derecho al proceso de desarrollo económico para lograr metas de desarrollo socioeconómico a través de instrumentos jurídicos, especialmente de derecho público, de funcionamiento del mercado. A partir de los fundamentos teórico-metodológicos de las relaciones entre el desarrollo económico y las instituciones jurídicas que establece la Nueva Economía Institucional, se superan las limitaciones del análisis 
del "movimiento del derecho y el desarrollo", y se establecen las bases para la construcción del Estado de Derecho promovente del desarrollo económico.

El Estado de Derecho de un país es el creador de un conjunto de incentivos que inciden en el desarrollo económico a través de un marco institucional para el control y la accountability del poder público. Por lo tanto, existe una relación de influencia recíproca directa entre el Estado de derecho y los niveles de desarrollo económico, social y político, aunque existen algunos análisis que tratan de explicar algunos impedimentos del desarrollo jurídico en un Estado de Derecho, el cual debe crear las condiciones para el ejercicio de los derechos civiles y político democráticos.

Desgraciadamente falta sistematización teórica para fundamentar el papel del derecho en el desarrollo económico, ya que solamente se fundamentaba en los trabajos de Weber sobre los análisis de modernización y en la jurisprudencia sociológica.

La literatura ha reportado las conexiones que existen entre el crecimiento económico y los determinantes de la democracia. Algunas investigaciones sugieren interacciones dinámicas positivas entre el desarrollo económico y el desarrollo político. Por un lado quienes realizaron estudios cuantitativos transversales-nacionales que tendían a coincidir con la ecuación optimista de Lipset (1979) que establecía que a mayor desarrollo económico de los pueblos, más oportunidades para la democracia, y por otro quienes realizaron estudios históricocomparativos.

Lipset (1979) ha dicho que cuanto mejor le va a un país en términos económicos, mayores son las oportunidades de mantener gobiernos democráticos. Las deficiencias de los procesos democráticos en el ámbito nacional son evidentes y de acuerdo a ciertos analistas, pueden ser subsanados mediante la creación de instituciones democráticas a mayor escala y conectándolas a las formas locales de participación.

Diamond (1992) reformula la ecuación optimista estableciendo que cuanto mejor le va a la gente de un país, en promedio, mayores son las oportunidades de que favorezcan, encuentren y mantengan un sistema democrático. Consecuente con esta línea de análisis, no existe un determinado nivel de desarrollo económico que sea determinante de la democracia (Przeworski y Limongi (1997). Las conclusiones de los analistas sobre la relación entre desarrollo económico y democracia son divergentes (ALTMAN, 2001). 
José Gpe. Vargas Hernández

Las dimensiones del cambio económico, político y social mundial están determinadas por la reestructuración del capitalismo globalizador. El capitalismo globalizador polariza el desarrollo económico y social de los pueblos. El capitalismo globalizador se encuentra amenazado por una espiral decreciente de crecimiento económico, es decir por una espiral decreciente de los ingresos per cápitas y por los promedios reales de consumo.

El desarrollo económico, político y social global comprende el equilibrio de todos los actores políticos y sociales y los agentes económicos. Es cuestionable considerar que el mercado es parte del ámbito de la sociedad civil, tomando en cuenta que ésta no constituye el Estado.

\section{Cambio y desarrollo económico}

El desarrollo económico determina la emergencia y sopervivencia de la democracia. Un elevado desarrollo económico limita el riesgo de que se abandone la democracia, aunque este mismo nivel de desarrollo en un sistema autocrático, no necesariamente implica que se abandone (Przeworski et al., 2000).

Williamson (1985) y North (1998 y 1990) resaltan la importancia del rol que las instituciones juegan en el desarrollo económico y sugieren que la evolución de las estructuras de gobernabilidad de las instituciones políticas y económicas genera ambientes que contribuyen al aumento de la productividad y el crecimiento económico. Un trabajo posterior de Campos (1999) sugiere que existe un grupo de variables institucionales fundamentales para la promoción del desarrollo económico, entre las que destacan la calidad de la burocracia y el Estado de Derecho.

El diseño institucional de un país es determinante para su desarrollo económico, social y político y condicionan las consecuencias del sistema político. Las instituciones políticas son el sustento del desarrollo económico y social. El mismo Banco Interamericano de Desarrollo (2000) reconoce que "la asociación entre calidad de las instituciones y desarrollo económico, humano y social, es especialmente estrecha, en parte porque las instituciones están influidas por el mismo proceso de desarrollo".

Entre el desarrollo y las instituciones existe una relación estrecha, la cual queda perfectamente manifiesta en Sen (2000) cuando señala que: "Los individuos vivimos y operamos en un mundo de instituciones de las que no somos siempre conscientes, muchas de las cuales trascienden hoy las fronteras nacionales. Nuestras oportunidades y 
perspectivas dependen en gran medida de las instituciones que existen y de cómo funcionan. Las instituciones no solo contribuyen a nuestras libertades, sino que deben ser evaluadas en su función de su contribución a nuestras libertades. Así lo exige el contemplar el desarrollo humano como libertad".

La gobernabilidad democrática implica procesos democráticos, la legitimidad y la regla de ley, que se logran a través de los procesos de desarrollo económico y social de los pueblos.

El mundo está en constante transformación y cambio económico. Mientras que muchos países están experimentando un crecimiento económico sostenido, otros todavia países todavía permanecen en la pobreza. Para mantener sus ventajas, los países desarrollados cancelan las oportunidades y prácticas que utilizaron para alcanzar altos niveles de crecimiento económico y aprovechan para continuar la explotación mediante la fórmula histórica de políticas para el desarrollo económico. Los sistemas económicos disfuncionales conducen a mecanismos perversos de crecimiento y desarrollo económico y por ende de desarrollo político.

El colapso de la economía socialista y la implosión estratégica de las economías de mercado que abandonan el modelo de desarrollo centrado en el Estado nación y basado en la industrialización por sustitución de importaciones, dieron lugar a una transformación cualitativa de los procesos de desarrollo económico impulsados por una competencia abierta entre localizaciones ubicadas en distintas partes del mundo, dando lugar a una globalización de los procesos económicos.

De acuerdo con Burgos Silva (2002), el análisis económico neoinstitucionalista define la institucionalidad jurídica y el desarrollo económico, cuestiona el derecho como instrumento del desarrollo económico y reconoce a las instituciones informales. además promueve mecanismos institucionales considerados como óptimos. De acuerdo con Prats (2002), "El mensaje del neoinstitucionalismo económico es una buena nueva para la democracia liberal: en las condiciones actuales las instituciones necesarias para definir y garantizar los derechos individuales requeridos para el mayor y mejor desarrollo económico no sólo son compatibles sino que son las mismas necesarias para disponer de una democracia duradera (OLSON, 1993).

El involucramiento cívico y las actitudes culturales dan forma al funcionamiento de las instituciones políticas mediante cierto capital social que puede ser adquirido en cierto tiempo (ALMOND and VERBA, 1963 y LIPSET, 1979). Las relaciones de confianza interpersonal, cooperación 
José Gpe. Vargas Hernández

y compromiso cívico aprendidas en asociaciones crean un capital social que influye en el desarrollo económico y el desempeño democrático de las instituciones (Putnam, 1993; Inglehart and Welzel, 2005 y Hadenius and Teorell (2005). Así, un elevado desarrollo económico puede coexistir con un debilitamiento de las relaciones de confianza y cooperación cívica (Grootaert, 1998).

Przeworski et al. (2000) concluyen que el crecimiento en el ingreso económico incrementa la sobre vivencia de la democracia pero no afecta la sobre vivencia de la autocracia

\section{Cambio y desarrollo político}

El desarrollo político o de gobierno ocurre en todas las instancias del sistema político y orienta los cambios con fundamentos en la democracia liberal. Los regímenes políticos dan forma dan forma al desarrollo económico. Sobre este punto, los resultados de las investigaciones empíricas se dividen. Por un lado quienes concluyeron que no hay efectos robustos de la democracia sobre el crecimiento económico (Londregan and Poole, 1990; Przeworski and Limongi, 1993; Helliwell, 1994 and Mulligan and Sala-I-Martin, 2004), y aquellos que encontraron resultados que apoyan un promedio positivo de los efectos de la democracia sobre los resultados económicos, aunque con heterogeneidad relevante en los episodios de democratización (ROLL and TALBOT, 2003; PAPAIOANNOU and SIOUROUNIS, 2004; JONES and OLKEN, 2005; GIAVAZZI and TABELLINI, 2005 and RODRIK and WACZIARG, 2005).

La historia democrática de un país tiene efectos en el desempeño económico (Gerrind, Bond, Barndt and Moreno, 2005). La democracia como sistema político influencia el desarrollo económico de los pueblos. Algunos estudios concluyen que la democracia acelera el crecimiento económico, aunque no existen muchas evidencias, otros estudios concluyen que no existe una conexión fuerte entre las dos variables. En las democracias estables, se acelera el crecimiento económico, lo cual a su vez, contribuye a aumentar la estabilidad democrática en una espiral de un circulo virtuoso. La estabilidad democrática es un factor importante para alcanzar más rápidamente el desarrollo económico.

Persson y Tabellini (2005) elaboraron un modelo teórico de cambio económico y político para demostrar que el régimen político actual y esperado influencia el crecimiento económico. La probabilidad del cambio de régimen depende del capital democrático nacional, el cual influencia 
la voluntad de los ciudadanos para luchar por la democracia. La identificación se consigue por una restricción de exclusión que consiste en que el capital democrático no tiene efectos directos en el crecimiento. De acuerdo con Persson and Tabellini (2006), la estabilidad democrática está determinada por una larga tradición de gobiernos democráticos y cuando se está rodeado de democracias que funcionan bien.

Un crecimiento más acelerado se asocia con democracias más estables y los efectos estimados de la democracia actual son más fuertes cuando la probabilidad de cambio de regimenes es constante (PERSSON and TABELLINI, 2006). Las expectativas sobre los cambios de regimenes políticos juegan un papel importante en el desarrollo económico. Así, las expectativas de estabilidad que generan las democracias contribuyen al crecimiento económico más que las expectativas de los regimenes autocráticos. El riesgo de que se abandone una democracia daña el crecimiento económico

Si se considera el orden político como una condición necesaria para el desarrollo económico, se reconoce que el sistema político garantiza los derechos de la ciudadanía que sirven de fundamento al modelo de crecimiento económico. Por lo tanto, el crecimiento económico requiere de un orden político que puede ser establecido en forma consensual, en forma autoritaria e incluso mediante procesos de desorden.

La sociedad autoritaria limita el alcance de un orden político basado en la cooperación social y desarrolla un sistema de intercambio político donde se otorgan derechos y privilegios por lealtad y apoyo. Las autocracias que se convierten en democracias continúan siendo inestables y vulnerables e imposibilitan el crecimiento económico. Sin embargo, Londregan y Poole (1990), no encontraron evidencia de los efectos de la inestabilidad política causada por los levantamientos políticos en el crecimiento económico.

La democracia no está interesada en la participación popular para el establecimiento de la agenda de desarrollo económico, político y social, sino más bien en buscar la legitimación del proyecto económico puesto en boga por los políticos. Esta situación ha dado lugar a la degradación de sistemas democráticos, tipificados como democracias delegativas, las cuales se han implantado en muchos países, comparado con los ya establecidos en las democracias europeas.

Las democracias delegativas, según O’Donnell (1994) simulan tener las características de una democracia liberal pero con instituciones 
José Gpe. Vargas Hernández

políticas débiles que son aprovechadas por los políticos que ejercen el poder con flagrante violación de los derechos humanos.

El clima político internacional impacta la estabilidad democrática por lo que la inestabilidad democrática tiene implicaciones en las transiciones de los regimenes políticos. La nueva gobernabilidad supranacional que se ejerce en las estructuras de poder internacionalizadas implica un orden basado en acuerdos negociados para la coordinación de políticas normativas y estrategias de desarrollo económico, social y político conjunto que supere el impulso cautivador de los grandes intereses que buscan sus ventajas y beneficios.

Los procesos de democratización representan un paso significativo para la resolución de problemas del desarrollo económico, social, político y la consolidación de la gobernabilidad mediante cambios profundos que implican la definición de los límites entre los sectores público y privado, principios y procesos de las funciones del Estado.

De acuerdo a Weaver y Rockman (1993).

(...) las respuestas que los países recientemente democratizados tengan ante los retos y desafíos que les plantea el desarrollo económico y la integración política y social de sus pueblos, dependerán en medida importante de la opción de diseño institucional que adopten.

La promoción de los procesos de democratización debe considerar las condiciones locales y asegurar su cumplimiento con actividades que benefician a los ciudadanos. Las funciones del Estado deben complementar los mercados mediante una coordinación de las interacciones de intercambio y movilizar los agentes de desarrollo económico de una sociedad. La estrategia de crecimiento económico se orienta hacia la descentralización de funciones del Estado, la participación y democratización. Sin embargo, los resultados de la implementación de estas reformas son decepcionantes: se incrementaron los niveles de inequidad y dualización social e incremento de la inestabilidad económica, política y social.

En los países donde el desarrollo político es volátil, se viven períodos intermitentes de democracia y autocracia. La inestabilidad política de una autocracia daña el crecimiento económico, sobretodo cuando se predicen cambio de régimen (PRZEWORSKI et al 2000). El 
cambio político democrático establece compromisos que instrumenta la solución de conflictos.

La teoría de la autoridad - cultura de Eckstein (1996) sostiene el papel dinámico de la cultura en los procesos de cambio político.

Para atender las diferencias locales, bajo un enfoque de federalismo, el Estado debe formular e implementar políticas diferenciales que consideren sistemas de incentivos a los comportamientos de los actores sociales y políticos que operen como articuladores del desarrollo económico social local para desactivar la relacion perversa existente entre la clase politica y el clientelismo.

\section{Capital democrático}

El capital democrático es la acumulación de activos sociales y cívicos que resulta de procesos de consolidación de aprendizajes de un país de su experiencia histórica y de sus países vecinos. El capital democrático se refiere a las variables que tienen influencia en la estabilidad de los regimenes democráticos pero que no tienen efectos directos en los resultados económicos (PERSSON y TABELLINI, 2006). El capital democrático se define como la forma en que los ciudadanos toman decisiones.

Ottaway (1999) denomina capital democrático a las condiciones de existencia colectiva de las sociedades, tales como la infraestructura colectiva, las tradiciones de la vida civil, las estructuras de autoridad efectivas y los mecanismos para la conciliación y la participación ciudadana.

Ottaway (1999) incorpora el concepto de capital social de Putnam en su concepto de capital democrático. Sin las adecuadas dotaciones de estas condiciones, las elecciones periódicas, la competencia de los partidos políticos, los medios de comunicación independientes, la economía de libre mercado, los mismos atributos de la democracia de los pueblos democráticos, de las políticas del gobierno, de las organizaciones no gubernamentales occidentales, serian proclives a acentuar las divisiones sociales, religiosas y étnicas de una sociedad, al menos a corto plazo.

Las organizaciones de la sociedad civil construyen el capital democrático que consiste en las habilidades concretas que son requeridas para formar una democracia moderna, permitiendo a las generaciones futuras de ciudadanos y a los futuros lideres del gobierno 
José Gpe. Vargas Hernández

aprender como planear, tomar decisiones, manejar reuniones y negociar fines comunes.

El capital democrático se mide por la acumulación en años de democracia y por los ambientes de los vecinos democráticos, pero se deprecian con experiencias autocráticas. El capital democrático medido por la experiencia histórica de la nación con la democracia y por su incidencia de democracia con sus vecinos, tiende a reducir la salida del sistema democrático y eleva la probabilidad de salir de sistemas autocráticos. Capital democrático y prosperidad económica viene aparejado con seguridad humana.

El capital democrático se forma por un componente doméstico y otro foráneo. El capital democrático doméstico depende de la propia experiencia histórica nacional, el cual se acumula en periodos democráticos y se desacumula en períodos de autocracia. El capital democrático doméstico es esencialmente una función del tiempo que tiene una sociedad de ser una democracia. El capital democrático foráneo depende de las influencias de las experiencias democráticas en otros países, y se desacumula con las experiencias foráneas autocráticas. La acumulación del capital democrático trae consigo un mayor crecimiento económico y una mayor estabilidad democrática. Una gran acumulación de capital democrático puede contribuir a que sucumban las autocracias.

En las democracias, una alta reserva de capital democrático estimula el crecimiento de forma indirecta reduciendo las posibilidades de levantamientos armados. El capital democrático y el capital institucional tienen como marco de referencia la totalidad de las instituciones que son relevantes para el análisis de los procesos del desarrollo económico y en general para las actividades económicas. Existe una relación significativa entre las democracias consolidadas, la confianza en las instituciones y el desarrollo económico y social.

Con respecto al capital democrático, Persson y Tabellini (2006) sugieren que su incremento no tiene un efecto directo en el crecimiento, solo un efecto indirecto en el incremento de las expectativas de retornos de inversiones. El capital democrático tiene un efecto positivo en el crecimiento económico. La acumulación de capital democrático tiene impactos tanto en la estabilidad democrática como en el incremento de los ingresos económicos.

El capital democrático de un pueblo se desarrolla en función de los hechos históricos, sistema político, derechos constitucionales, responsabilidades públicas, las leyes que regulan la corrupción, los procesos legislativos y judiciales, etc. El incremento notable en los últimos 
años de las organizaciones sociales y las organizaciones no gubernamentales, consolidan la participación activa de los movimientos de liberación, de derechos humanos y de democratización, en amplios programas de desarrollo económico, político y social.

La participación en los procesos políticos de las organizaciones sociales mediante arreglos institucionales que promuevan su democratización y garanticen al Estado su capacidad de regulación, permitirá a estas organizaciones su participación en la formulación e implantación de políticas públicas. El orgullo cívico es un elemento vital de renovación para el Estado moderno. Las relaciones de confianza interpersonal, cooperación y compromiso cívico aprendidas en asociaciones crean un capital social que influye en el desarrollo económico y el desempeño democrático de las instituciones.

Las tecnologías de la información y la comunicación (TIC) son vistas como nuevas formas de organización social y como procesos de transformación y cambio económico, político, social, cultural, etc., mediante la formación de comunidades u organizaciones de protesta y la emergencia de movimientos con nuevas inquietudes y reivindicaciones.

En su reporte Price Waterhouse Coopers sugiere como ingredientes para hacer las naciones más competitivas: Capital social e intelectual, capital democrático, capital cultural y de ocio, capital ambiental y técnico y capital financiero.

La tolerancia a las diferencias, la pluralidad y diversidad de las manifestaciones y movimientos políticos y sociales son parte del capital democrático que sirve de base a la unidad de las fuerzas de cambio y de transformación y crea espacios de diálogo y concertación. En estos espacios que ejercitan el aprendizaje de la tolerancia y el respeto a la diversidad de ideas, se genera una cultura democrática y se logra una acumulación del capital democràtico.

Algunas naciones, como la India, han desarrollado capital democrático después de su Independencia como un recurso de legitimidad y de identidad, el cual es representado por una democracia constitucional, liberal pero no en su forma mayoritaria. Este dividendo democrático coloca a la India de estar preparada para la batalla global de las ideas.

Al mismo tiempo que se redefinen los poderes económico y político transnacionales, el modelo de desarrollo Indio está demostrando que la India es capaz de incrementar su poder económico conciliado con su habilidad para nutrir su diversidad y pluralismo interno a través de 
José Gpe. Vargas Hernández

estructuras de democracia liberal constitucional (MISHRA, 2006). La India se ha dado cuenta de que su capital democrático construido en las últimas décadas del siglo pasado tiene un valor instrumental enorme en un ambiente donde las batallas de imágenes y las ideas se vuelven más feroces.

Por el contrario, en Rusia, el poder económico de las organizaciones criminales que han resultado de los procesos de transición de sistema económico no permite el desarrollo de capital democrático plenamente.

Martínez (2003) argumenta que del stock de capital democrático que es de 60\% en dieciocho países de la región latinoamericana, de acuerdo con datos proporcionados por el Programa de las Naciones Unidas para el Desarrollo (PNUD) la mitad estaría dispuesta a aceptar un régimen autoritario si trajera solución a sus problemas económicos. El PNUD, propone una política generadora de poder democrático que tenga como objetivo formar a la ciudadanía integral capaz de afrontar el problema de la pobreza y la exclusión social, para que la persona acceda armoniosamente a sus derechos cívicos, sociales, económicos y culturales y revertir la devaluación del capital democrático de la región.

La investigación del PNUD ha sido corroborada por otras, como Latino barómetro, las cuales confirman que la realidad en Latinoamérica es muy diferente a las conclusiones a que llegan las investigaciones sobre capital democrático.

Las iniciativas de muchos presidentes latinoamericanos, por ejemplo, se dan en un contexto con más capital democrático de lo que están dispuestos a reconocer muchos de los críticos del régimen político. La experiencia de los Uribistas en Colombia y de Transparencia en Perú como espacios que acumulan capital democrático, y como repositorios de conocimientos teóricos y prácticos sobre los temas que definen los grandes desafíos que enfrenta el ejercicio de la democracia en esos países. El capital democrático de una nación se deposita y reside en sus instituciones y en su capacidad para dar soluciones a los conflictos de interés, que lamentablemente muchos pueblos no logran, como es el caso de Perú con Fujimori y de los fracasos de Uribe para forjar un régimen autoritario fuerte en Colombia.

Lo que se requiere es un enfoque a las causas de las desilusiones mediante la formulación e implantación de estrategias coherentes con una visión de sociedad democrática que mediante procesos políticos reconstruyan la confianza entre las personas y que contribuyan a la formación de capital democrático. 
Las tendencias que guían al capital democrático en los procesos de tomas de decisiones consideran las interacciones entre los ciudadanos de una sociedad y las influencias de las fuerzas económicas y políticas, con el alineamiento de sus respectivos objetivos.

Las tendencias actuales para la formación de capital democrático apuntan lograr una mejor transparencia y comunicación, el desarrollo de asociaciones entre los sectores públicos y privados y una mayor participación.

El balance y la calibración de las diferentes formas de participación democrática es un factor para la optimización del capital democrático. La colaboración y participación ciudadana en los procesos de tomas de decisiones públicas son esenciales cuando las tendencias globales apuntan en direcciones opuestas: hacia un individualismo egoísta y su concomitante declinación en los valores sociales compartidos, apatía política, etc.

Para Assad (2006) el capital democrático significa que existen procesos de participación y consultación por el público lo que por consiguiente prueba que hay transparencia pública y rendición de cuentas (accountability). El capital democrático desarrolla la cultura de la participación ciudadana. Cuando el stock de capital democrático es abundante, los ciudadanos son más participativos en los procesos de tomas de decisiones que afectan a sus comunidades. El capital democrático se utiliza para dirimir las diferencias y alcanzar compromisos legítimos en base a las reglas de la negociación política.

El capital democrático es un sistema de libertad natural que forma las bases de una comunidad genuina (YOUNKINS, 1999). El capital democrático usado para el bien común es esencial para el desarrollo de organizaciones sociales y comunidades. La acumulación de capital físico y democrático se refuerza mutuamente, pues promueven el desarrollo económico al mismo tiempo que se consolida la democracia. 
José Gpe. Vargas Hernández

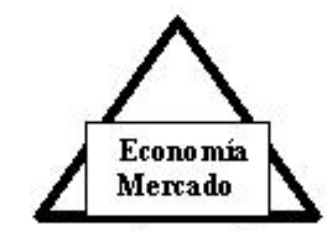

Grandes corporaciones, mic ros, pequeñas y medianas emp resas Comunidades bcales de negocios,

Cámaras, etc.

Organizaciones p rofesionales

Gran tendencia 1

TRANSPARENCI
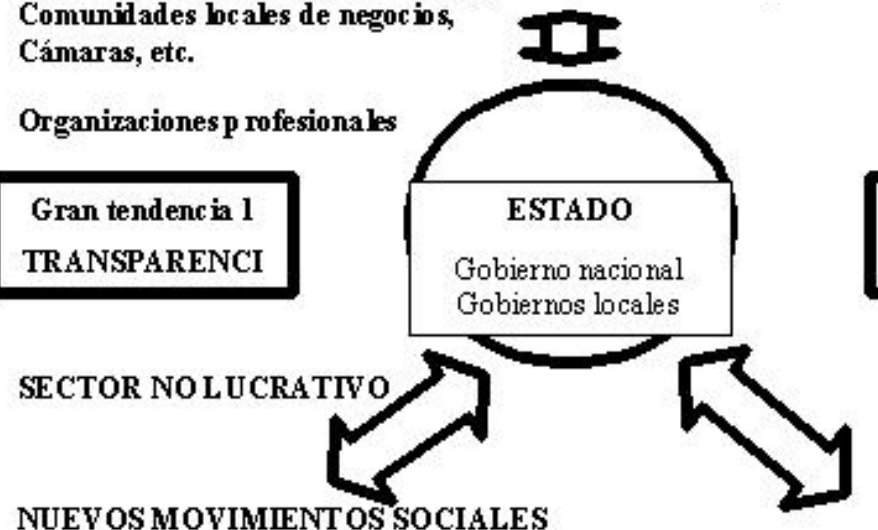

Sindicatos

NUEVOS MOVIMIENT OS SOCIALES GRUPOS DE PRESION

Organizaciones no gubernamentales (ONGs)

Partidos políticos

Vida del club social

Redes informales de vecindades en el trabajo

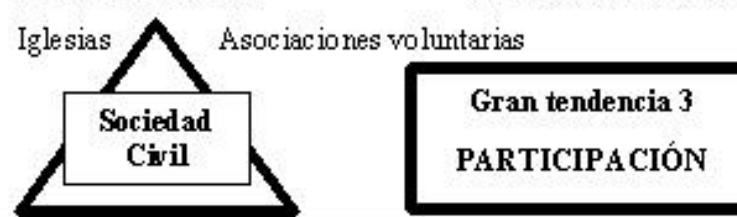

COMUNIDAD

Familas, haso de amistad

Figura 1: La sociedad civil y sus relaciones con la comunidad, el mercado y el Estado. Interacciones entre la política, la sociedad y la economía con las tres mayores tendencias para optimizar el capital democrático. 


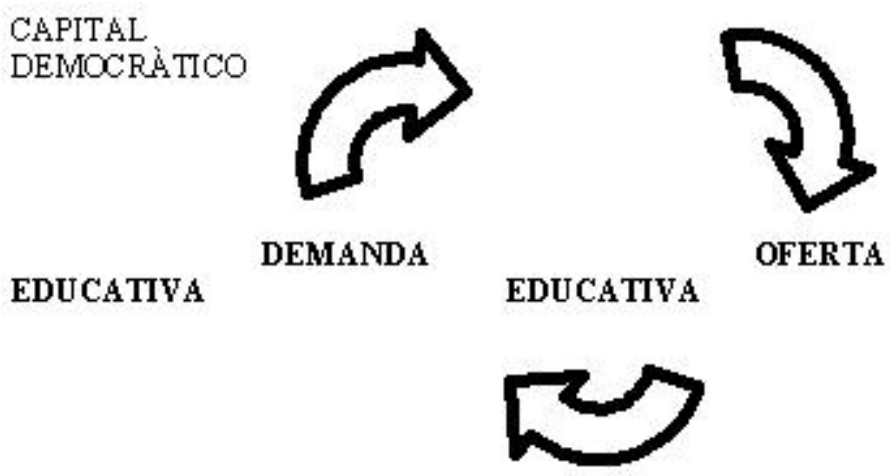

Figura 2: Relaciones entre el capital democrático de una nación con la oferta y la demanda educativa.

Fonte: Adopted from Levinger, Beryl (2006).

Para poner un ejemplo de cómo este modelo se aplica, en la breve experiencia de la Rusia democrática, los niños están recibiendo educación forzada de conceptos sociales elementales sobre la participación en los procesos democráticos. Las escuelas públicas de Chicago implementan una propuesta a largo plazo de proceso de planeación para la formación de capital democrático.

El capital democrático se forma con la aplicación sistemática de procesos de producción los cuales dan forma a la oferta y demanda educativa y están siempre en relación con los resultados asociados. Por lo tanto, se presentan relaciones dinámicas entre los procesos de producción de capital democrático y los resultados asociados con la formación de capital democrático, los cuales a su vez pueden ser las fuerzas primeras que determinan los procesos.

\section{Discusión}

Los Estados nacionales presentan diferencias profundas en sus niveles de desarrollo económico y social, en niveles de riqueza y sus valores, etc., como para que tengan las mismas consideraciones con respecto al sistema democrático. Los valores económicos y tecnológicos son importantes, pero es igualmente esencial el desarrollo del capital democrático y cívico de la sociedad.

North (1991) argumenta que la teoría macroeconómica nunca resolverá los problemas que confronta a menos que reconozca que las decisiones adoptadas en el proceso político afectan críticamente el 
José Gpe. Vargas Hernández

funcionamiento de la economía, lo cual puede hacerse mediante una modelización del proceso económico-político que incorpore las instituciones específicas afectadas y la consiguiente estructura del intercambio político y económico.

La nula o poca convergencia entre el mercado y la democracia permite concluir que el desarrollo económico por medio de los mercados difícilmente es compatible con grados avanzados de democracia. El Estado puede balancear con el mercado el desarrollo económico. La formulación de políticas sociales que alientan las capacidades sociales y humanas para el desarrollo económico previene la erosión de valores sociales fundamentales y mueven a las sociedades hacia un desarrollo más equitativo y sustentable que requiere más que simplemente ajustar los enfoques económicos y sociales.

El movimiento de la gente se sustenta en ideas que la divide entre las personas y las corporaciones multinacionales, entre el capital democrático y el poder del capital corporativo. Solo que este planteamiento va más allá que la teoría marxista que enfrenta a la clase trabajadora con la burguesía capitalista.

El capital democrático irreducible de una sociedad determinada puede manifestarse en una absoluta indiferencia con su entorno político, como en el caso del abstencionismo electoral. La democracia no se resuelve solamente en el juego de las representaciones políticas. 


\section{REFERÊNCIAS}

ALTMAN, David (2001b). "Democracia política y desarrollo económico: ¿Existe alguna realidad? Magazine No. 18, Instituto Internacional de Gobernabilidad, Junio, 2001. http://www.iigov.org/iigov/pnud/ bibliote/dhial/dhial18_05.htm.

ALMOND, Gabriel, and Verba, Sydney. The civic culture: Political attitudes and democracy in five nations. Princeton University Press. 1963.

ASSAD, Nasser Al. "New strategic vision for Amman" The Star, June 8-14, 1 Issue 110. 2006.

Banco Interamericano de Desarrollo. Desarrollo más allá de la política. Washington, D.C. . BID, 2000.

BURGOS SILVA, Germán. "Estado de Derecho y desarrollo económico: aportes y limitaciones de los análisis neinstitucionales", Revista Instituciones y Desarrollo No. 12-13, páginas 193-221. Institut Internacional de Governabilitat de Catalunya, Barcelona. 2002.

CAMPOS, N. Context is everything: measuring institutional change in transition economies. Working Paper. CERGE-EI, Czech Republic. 1999.

DIAMOND, L. "Economic development and democracy reconsidered." American Behavioral Scientist 35:450-499. 1992.

ECKSTEIN, Harry. Lessons for the "third wave" from the first. Center for the study of democracy, UC Irvine: Research Paper Series in Empirical Democratic Theory, No. 2. 1996.

GERRIND, John; Bond, Philip; Barndt, William T. and Moreno, Carola, "Democracy and economic growth: A hostorival perspective", World Politics, 57, 323-354. 2005.

GIAVAZZI, Francesco and Tabellini Guino. "Economic and political liberalizations", Journal of Monetary Economics 52, 1297-1330. 2005. 
José Gpe. Vargas Hernández

GROOTAERT, Christiaan. "Social capital: the Missing Link?" Social Capital Initiative. Paper 3, World Bank, April, 1998.

HADENIUS, Axel and Teorell, Jan. "Cultural and economic prerequisites of democracy: Reassessing recent evidence", Studies in comparative International Development 39, 87-206. 2005.

HELLIWELL, John. "Empirical linkages between democracy and economic growth", British Journal of Political Science 24, 225-248. 1994.

INGLEHART, Ronald and Welzel, Christian. Modernization, cultural change and democracy: The human development sequence, Cambridge University Press. 2005.

JONES, Ben and Olken, Benjamin. "Do leaders matter? National Leadership and growth since World War II", Quarterly Journal of Economics 120, 835-864. 2005.

LEVINGER, Beryl. Education, democracy, and the building of a common future. Global Learning Group. Education Development Center. 2006.

LIPSET, Seymour Martin. "Some social requisites of democracy: Economic development an political legitimacy", American Political Science Review, 53, 69-105. 1979.

LONDREGAN, John and Poole, Keeith. "Poverty, the coup trap and the seizure of executive power", World Politics 42, 151-183. 1990.

MARTINEZ, Elena. Financiamiento de la democracia: Intervención de Elena Martínez, News \& Info. The Carter Center. 2003.

MISHRA, Vandita. "The vote for India", The Indian Express. June 14, 2006.

MULLIGAN, Casey B., Sala-i-Martin, Xavier and Gil, Ricard. "Do democracies have different public policies than nondemocracies?" Journal of Economic Perspectives 18, p. 51-74. 2004. 
NORTH, D. La teoría económica neoinstitucionalista y el desarrollo latinoamericano. Proyecto PNUD "Red para la Gobernabilidad y el Desarrollo en América Latina- Instituto Internacional de Gobernabilidad. Barcelona, España. 1998.

NORTH, D.D. Institutions, Institutional Change and Economic Performance, Cambridge, Cambridge University Press. 1991.

NORTH, Douglas. "A transaction cost theory of politics," Journal of Theorethical Politics, 2 (No. 4): 355-367. 1990.

NOVAK, Michael. The Catholic Ethic and the Spirit of Capitalism (New York: The Free Press). 1993.

O'DONNELL, Guillermo. "Delegative democracy", Journal of democracy. Vol. 5, no. 1, p. 55-69. 1994.

OLSON, Mancur. "Dictatorship, Democracy and Development", en American Political Science Review, vol. 87, número 3, septiembre. 1993.

OTTAWAY, Marina. Africa's New Leaders: Democracy or State Reconstruction? Washington, DC: Carnegie Endowment for International Peace, 1999. viii + p. 138. 1999.

PAPAIOANNOU, Elias and Siourounis, Gregorios. "Democratization and growth", Mimeo, LBS. 2004.

PERSSON, Torsten and Tabellini. Democratic capital: The nexos of political and economic change. Working Paper 12175. Working Paper Series. National Bureau of Economic Research, Inc. 2006.

PERSSON, Torsten and Tabellini, Guido. "Democracy and Development: Devil in the Details". Mimeo. December 2005.

Price Water House Cooper. Cities of the future, global compeition, global leadership. Connectedthinking. Captured 26 of June, 2006. http:// www.pwc.com/py/spa/pdf/ciudades_futuro.pdf 
José Gpe. Vargas Hernández

PRZEWORSKI, Adam and Limongi, Fernanddo. "Political regimes and economic growth", Journal of Economic Perspectives 7, p. 51-69. 1993.

PRZEWORSKI, Adam, Alvarez, Michael, Cheibub, Jose, and Limongi, Fernando. Democracy and development: Political institutions and Well being in the World 1950-1900, Cambridge University Press. 2000.

PUTNAM, Robert. Making democracy work: Civic traditions in modern Italy, Princeton University Press. 1993.

RODRIK, Dani and Wacziarg, Romain. "Do democratic transitions produce bad economic outcomes?", American Economic Review Papers and Proceedings 95, p. 50-56. 2005.

ROLL, Richard and Talbott, John. "Political and economic freedoms and prosperity. Mimeo, UCLA. 2003.

RUSTOW D. "Transitions to democracy." Comparative politics 2-3 (April): p. 337-363. 1970.

SEN, Amartya. El desarrollo como libertad. Barcelona: Paidos. 2000.

WEAVER R. Kent y Rockman, A. (eds.). Do institutions matter? Government capabilities in the United States and Abroad. The Brookings Institution, Washington, DC. 1993.

WILLIAMSON, O. The Economic Institutions of Capitalism: Firms, markets, relational contracting. Free Press. New York. 1985.

YOUNKINS, Edward W. "Michael Novak's Portrait of Democratic Capitalism". Journal of Markets \& Morality 2, no. 1(Spring 1999), p. 8-34. 1999. 